\title{
Effect of Dietary Fibers on Blood Lipid Parameters in Hypertension Patients of Indore District
}

\author{
Khurana $\mathrm{N}^{1}$, Dhakad $\mathrm{N}^{2}$ \\ ${ }^{I}$ Deptt. Of biotechnology, holkar science college, Indore, INDIA, \\ ${ }^{2} A d d$. Director of higher education, Indore Ujjain division, INDIA.
}

\begin{abstract}
Each year, over three million deaths worldwide are tied directly to diabetes and even greater number die from cardiovascular disease. Modification in lifestyle and proper medication can delay and prevent cardiovascular diseases in high-risk groups. Eating whole grain carbohydrates and moderate exercise and avoiding excessive weight gain could eliminate over eighty per cent of cardiovascular diseases. The SITE study, sponsored by a multinational pharmaceutical company, Sanofi, tested almost 16,000 people living in urban cities across eight states over the last three years. It concluded that one in every five Indian adults living in urban cities suffers from hypertension and diabetes. Your blood pressure is the force exerted on your artery walls by the blood flowing through your body. A blood pressure reading provides two measures: Systolic Pressure and Diastolic Pressure .Both are expressed as millimeters of mercury (mm Hg).Systolic pressure is measured as the heart pumps. Diastolic pressure is measured between beats, as blood flows back into the heart. High blood pressure is often called the "silent killer" because it has no symptoms and can go undetected for years. It is important to have your blood pressure checked regularly. Dietary fiber (alternate names: roughage, bulk, diet-fiber) is a complex of substances of cell walls of plants which are not digested and not absorbed in a human's digestive tract. There are two major kind of dietary fibers Insoluble and Soluble type. Soluble and insoluble fiber act in totally different ways. Eating whole grain carbohydrates and moderate exercise and avoiding excessive weight gain could eliminate over eighty per cent of cardiovascular disease. This study is a clinical Trial with 40 non-smoker Patients which had no renal and liver disorder. The patients were selected through questionnaire data collection method, by ways of various health care camps organized in the city. They were randomly divided into 2 groups; the first group was given 30-45 grams of dietary fibers per day (intervention group). The control group remained normal with no additional dietary supplements. All the groups were observed for a period of 2 years. Lipid parameters viz. HDL, LDL, TOTAL CHOLESTROL, TG was measured every year during the study for each group.Mean and SD were calculated. The significance of differences in the means of intervention group and control group were found using $t$ test both paired and unpaired.
\end{abstract}

Keywords: cardiovascular disease, SITE study, Systolic Pressure, Diastolic Pressure, Lipid parameter, Dietary fiber $s$.

\section{Introduction:}

High blood pressure is often called the silent killer because in the initial stages it presents with no symptoms. It is only after an organ in the body is irritated or damaged, that the consequences of high blood pressure are realized. There are two major types of hypertension. Primary or essential hypertension that has no known cause, and is diagnosed in the majority of people. Secondary hypertension is often caused by reversible factors, and is sometimes curable. Cardiovascular diseases caused 2.3 million deaths in India in the year 1990; this is projected to double by the year 2020. Hypertension is directly responsible for $57 \%$ of all stroke deaths and $24 \%$ of all coronary heart disease deaths in India. Indian urban population studies in the mid1950s used older WHO guidelines for diagnosis (BP 160 and/or $95 \mathrm{mmHg}$ ) and reported hypertension prevalence of $1.2-4.0 \%$. Subsequent studies report steadily increasing prevalence from $5 \%$ in $1960 \mathrm{~s}$ to $12-15 \%$ in 1990s. Hypertension prevalence is lower in the rural Indian population, although there has been a steady increase over time here as well. Various studies have shown that the high incidence of Cardiovascular diseases in India is mainly because of sedentary lifestyle, lack of physical activity, obesity, stress and consumption of diets rich in fat, sugar and calories. This study is a clinical Trial with 40 non-smoker Patients which had no renal and liver disorder for a period of 2 years, to see whether change in lifestyle particularly adapting to a diet rich in dietary fiber can lead to decrease in blood sugar levels.

\section{Materials and method:}

This study is a clinical Trial with 40 non-smoker Patients which had no renal and liver disorder. These were again divided into two groups, intervention group with 30 patients and control group with 10 patients each. 
The patients were selected through questionnaire data collection method(see annexure), by ways of various health care camps organized in the city, via diabetic and hypertensive care camp organized by MA vashno group in mathlani garden, Indore; launch party of dietary fiber rich flour SIFER, through the reference of various diabetologist and cardiologist etc. The questionnaire was given to patients in order to inquire about their present status of disease and health and calculation of BMI. Weight was measured while the subjects were minimally clothed without shoes using digital scales and recorded to the nearest $0.1 \mathrm{~kg}$. Height was measured in a standing position without shoes using a tape meter while the shoulders were in a normal state. Through the help clinical dieticians diet charts were prepared for these patients after energy calculation. Each of these diet charts included fiber rich products in the range of 36-45 Gms of fiber per day. They were randomly divided into 2 group's intervention and control group. The first group consisted of 26 males and 4 females with average age of 49.5. While the control group consisted of 8 males and 2 females having an average age of 46.3. The first group was given 36-45 grams of fiber per day being included in various recepies. The control group remained normal with no additional dietary supplements. All the groups were observed for a period of 2 years. Lipid parameters viz. HDL, LDL, TOTAL CHOLESTROL, TG was measured each year of the study for each group. Mean values and SD were calculated. The significance of differences in the means of intervention group and control group were found using t test.

\section{Data And Its Analysis:}

Table 1

\begin{tabular}{|c|l|l|}
\hline \multicolumn{3}{|c|}{ Mean values intervention group } \\
\hline mean & Before & after \\
\hline hdl-c & 43.36666667 & 44 \\
\hline ldl-c & 174.0666667 & 163.8666667 \\
\hline total-c & 257.6066667 & 247.0133333 \\
\hline $\operatorname{tg}$ & 200.8666667 & 195.7333333 \\
\hline ratio(h/l) & 0.249635533 & 0.269283932 \\
\hline ratio(l/h) & 4.063173859 & 3.769072238 \\
\hline
\end{tabular}

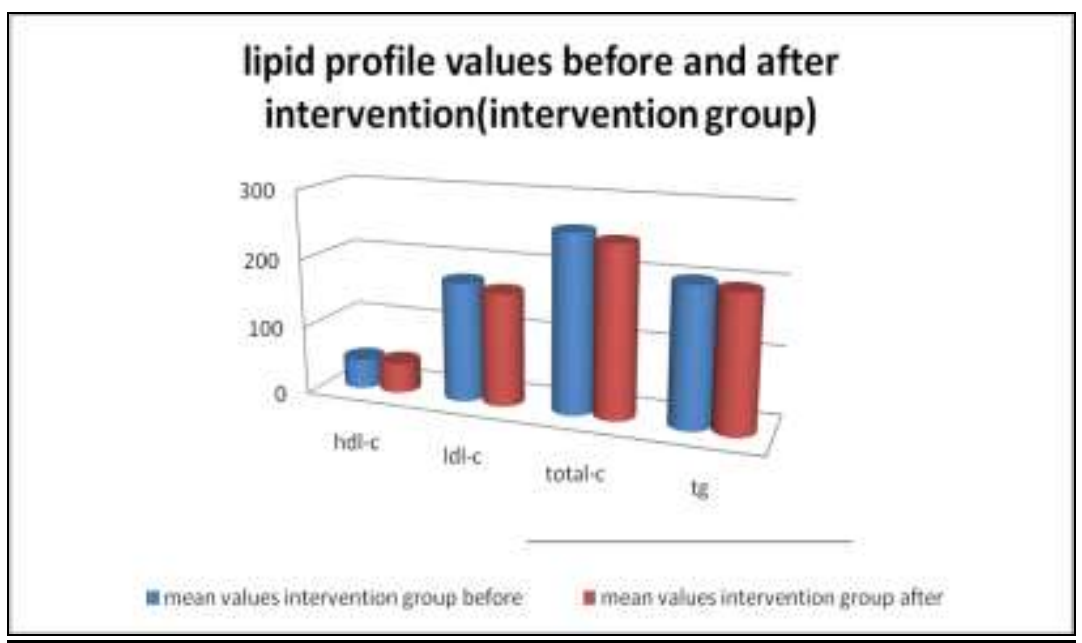

Figure 1

Table 2

\begin{tabular}{|l|r|r|}
\hline \multicolumn{3}{|l|}{ Mean values control group } \\
\hline Mean & before & \multicolumn{1}{l|}{ after } \\
\hline hdl-c & 40.2 & 40 \\
\hline ldl-c & 177.1 & 177.2 \\
\hline $\mathrm{Tg}$ & 225.1 & 224.9 \\
\hline Vldl & 45.02 & 44.98 \\
\hline $\begin{array}{l}\text { total } \\
\text { cholesterol }\end{array}$ & 262.32 & 261.78 \\
\hline ratio h/l & 0.227623 & 0.226 \\
\hline ratio $1 / \mathrm{h}$ & 4.467639 & 4.48 \\
\hline
\end{tabular}




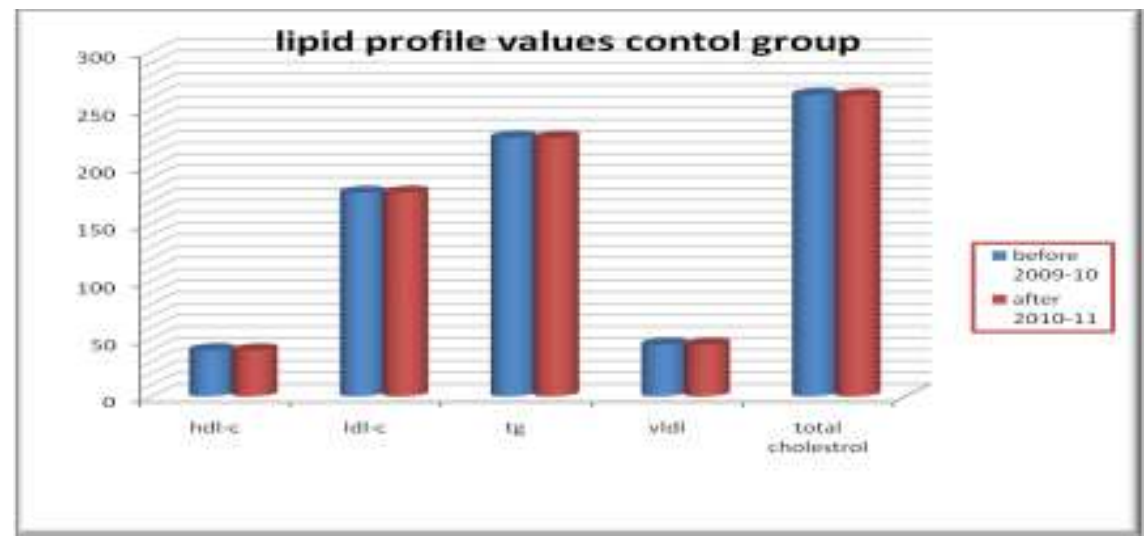

Figure 2

IV. Result And Discussion

As we can see from the above data that there is a big difference between the mean values of blood lipid parameters in intervention group (group given additional dietary fiber). Therefore in order to confirm that the difference is significant a paired $t$ test was applied. For the above values the calculated standard error and the calculated value of paired $t$ test for different parameters were found to be :

Table 3

\begin{tabular}{|l|l|l|}
\hline Parameter name & Standard error & Value of paired $\mathrm{t}$ \\
\hline HDL-CHOLESTROL & 0.251356 & -2.51966 \\
\hline TG & 0.981125 & 5.232091 \\
\hline LDL-CHOLESTROL & 1.285999 & 7.931578 \\
\hline VLDL-CHOLESTROL & 0.196225 & 5.232091 \\
\hline TOTAL CHOLESTROL & 1.293822 & 8.187628 \\
\hline
\end{tabular}

$\mathrm{H}_{0}=$ there is no significant difference between the mean of after supplementation and before supplementation in the intervention group.

$\mathrm{H}_{\alpha}=$ there is significant difference between the mean of after supplementation and before supplementation in the intervention group.

The table value for $\mathrm{t}$ at $5 \%$ level of significance and d.f $=29$ is 0.68 AND therefore the null hypothesis was rejected for every lipid parameter except HDL-CHOLESTROL. So there exists a significant difference between the mean values of lipid parameters in the intervention group except HDL-CHOLESTROL. Which is a good sign, i.e. dietary fibers do not affect good cholesterol but tends to decrease other cholesterol levels. Similarly to determine the decrease in lipid parameters in case of intervention groups was because of change in diet i.e. introduction of dietary fibers an unpaired t test was applied.

$\mathrm{H}_{0}=$ there is no significant difference between the mean's of intervention and control groups. $\mathrm{H}_{\alpha}=$ there is a significant difference between the mean's of intervention and control groups.

For the above values the calculated standard error and the calculated value of unpaired t test for different parameters were found to be

Table 4

\begin{tabular}{|l|l|l|}
\hline Parameter name & Standard error & Value of unpaired $\mathrm{t}$ \\
\hline HDL-CHOLESTROL & 32.7048 & 42.77082 \\
\hline TG & 37.04824 & 189.6629 \\
\hline LDL-CHOLESTROL & 14.33213 & 0.477738 \\
\hline VLDL-CHOLESTROL & 33.85112 & 37.81791 \\
\hline TOTAL CHOLESTROL & 45.72967 & 241.2801 \\
\hline
\end{tabular}

The table value for $t$ at $5 \%$ level of significance and d.f $=38$ is 2.02 AND therefore the null hypothesis was rejected for every lipid parameter except LDL-CHOLESTROL. So there exists a significant difference between the mean values of lipid parameters between the intervention groups and control group except LDLCHOLESTROL. Therefore Dietary fibers are highly effective in controlling cardiovascular disorders. 


\section{References:}

[1] Jenkins, David JA, et al. "Effect of wheat bran on glycemic control and risk factors for cardiovascular disease in type 2 diabetes." Diabetes Care 25.9 (2002): 1522-1528.

[2] Lecumberri, Elena, et al. "A diet rich in dietary fiber from cocoa improves lipid profile and reduces malondialdehyde in hypercholesterolemic rats." Nutrition 23.4 (2007): 332-341.

[3] Riccardi, Gabriele, and Angela A. Rivellese. "Effects of dietary fiber and carbohydrate on glucose and lipoprotein metabolism in diabetic patients." Diabetes Care 14.12 (1991): 1115-1125.

[4] Rivellese, Angela A., et al. "Long-term effects of fish oil on insulin resistance and plasma lipoproteins in NIDDM patients with hypertriglyceridemia." Diabetes Care 19.11 (1996): 1207-1213.

[5] http://articles.timesofindia.indiatimes.com/2011-12-14/health/30373026_1_blood-pressure-hypertension-site-study. 